\title{
Long-term visual and treatment outcomes of whole-population pre-school visual screening (PSVS) in children: a longitudinal, retrospective, population-based cohort study
}

\author{
Una O'Colmain ${ }^{1} \cdot$ Yan Ning $\mathrm{Neo}^{2} \cdot$ Claire Gilmour $^{3} \cdot$ Caroline J. MacEwen $^{4}$
}

Received: 8 March 2019 / Revised: 24 October 2019 / Accepted: 13 November 2019 / Published online: 25 February 2020

(c) The Author(s), under exclusive licence to The Royal College of Ophthalmologists 2020

\begin{abstract}
Background This study reports the long-term visual and treatment outcomes in a whole-population, orthoptic-delivered preschool visual screening (PSVS) programme in Scotland and further examines their associations with socioeconomic backgrounds and home circumstances.

Methods Retrospective case review was conducted on 430 children who failed PSVS. Outcome measures included best corrected visual acuity (BCVA), severity of amblyopia (mild, moderate and severe), binocular vision (BV) (normal, poor and none), ophthalmic diagnosis and treatment modalities. Parameters at discharge were compared to those at baseline and were measured against the Scottish index of multiple deprivation (SIMD) and Health plan indicator (HPI), which are indices of deprivation and status of home circumstances.

Results The proportion of children with amblyopia reduced from 92.3\% (373/404) at baseline to 29.1\% (106/364) at discharge $(p<0.001)$. Eighty percent $(291 / 364)$ had good BV at discharge compared to $29.2 \%(118 / 404)$ at baseline $(p<0.001)$. Children from more socioeconomically deprived areas (OR 2.19,95\% CI 1.01-4.30, $p=0.003$ ) or adverse family backgrounds (OR 3.94, 95\% CI 1.99-7.74, $p=0.002$ ) were more likely to attend poorly and/or become lost to follow-up. Children from worse home circumstances were five times more likely to have residual amblyopia (OR 5.37, 95\% CI 3.29-10.07, $p<0.001$ ) and three times more likely to have poor/no BV (OR 3.41, 95\% CI 2.49-4.66, $p<0.001$ ) than those from better home circumstances.

Conclusions Orthoptic-delivered PSVS is successful at screening and managing amblyopia. Children from homes requiring social care input are less likely to attend and are more likely to have poorer visual outcomes.
\end{abstract}

These authors contributed equally: Yan Ning Neo, Claire Gilmour, Caroline J. MacEwen

Una O'Colmain

uocolmain@nhs.net

1 Department of Ophthalmology, Ninewells Hospital and Medical School, Dundee, Scotland, UK

2 Moorfields Eye Hospital, Moorfields Eye Hospital NHS Foundation Trust, 162 City Road, EC1V 2PD London, UK

3 Department of Orthoptics, Ninewells Hospital and Medical School, Dundee, Scotland, UK

4 Department of Ophthalmology, Ninewells Hospital and Medical School, Dundee, Scotland, UK

\section{Introduction}

Amblyopia is the commonest vision deficit in children in the United Kingdom and is recognised to negatively impact the development of binocular vision (BV) and stereopsis [1-4].

The pre-school milestone (age 4-5 years) is considered the most effective time to perform vision screening [5, 6]. Binocular function develops from the age of 3-4 months and fully matures by the age of 8-9 years [7]. Although amblyopia screening is recommended by the National Screening Committee and the Hall (Four) Report $[8,9]$, its implementation has not been without considerable variation in terms of delivery policies, screening uptake and diagnostic pathways across the United Kingdom [10, 11]. In view of the heterogeneity of existing screening programmes and scarcity of evidence on treatment outcomes, there is a need for population-based studies of long-term screening outcomes [3, 11, 12]. 
The pre-school visual screening (PSVS) in Tayside is a whole-population orthoptic-delivered programme for 4 to 5 year-old children. Previously we reported the increased likelihood of failing screening for children who are socioeconomically deprived and those who come from high-risk homes where social care input is required [13]. The aim of this current study is to report the long-term visual outcomes of these children and to examine these with regard to socioeconomic and family circumstances.

\section{Methods}

\section{Setting and study design}

Details of the PSVS offered across Tayside, East of Scotland were reported in our previous study [13]. Screening is delivered by orthoptists and when a child fails screening, he or she is referred for repeat orthoptic assessment, cycloplegic refraction and fundus examination. The vision standard to pass PSVS is best-corrected visual acuity (BCVA) of $\leq 0.2 \log$ MAR on the crowded Keeler test with each eye, or $\leq 0 \cdot 1 \log$ MAR with crowded Kay pictures, if letter testing is not achieved. Children with significant refractive error are prescribed glasses and reviewed in the orthoptic clinic after up to 16 weeks; amblyopia therapy, if required, includes occlusion or atropine penalisation. Children who are treated for amblyopia are examined every 6-8 weeks until BCVA improves to an age-appropriate level or is stable and deemed unlikely to improve further.

The study group comprised the same 523 children who failed PSVS from a total number of 4365 (11.9\%) children screened between March 2010 and February 2011 (as in our previous study) [13]. A retrospective case review was performed to identify visual outcomes for each child up until either their final discharge visit, or most recent outpatient visit whichever came later. Outcome measures included BCVA, refractive status, residual amblyopia (if any) and BV. As we have previously reported on the rate of screening uptake and reasons for failing screening, these are not included in our current report [13]. In the event when a child had bilateral amblyopia, data from the worse seeing eye was used to avoid inter-eye correlations. Given the study was not conducted in a trial setting, there is no standard operating procedures for orthoptic appointments as the orthoptists work as autonomous practitioners who pick the most appropriate test for examination depending on the child's level of cooperation and vision on the day of visit.

Ninety-three children either did not attend any clinic appointments after the screening event or no follow-up data were available, leaving 430 children with clinical information on both their screening and subsequent follow-up appointments. Children who failed to attend were offered

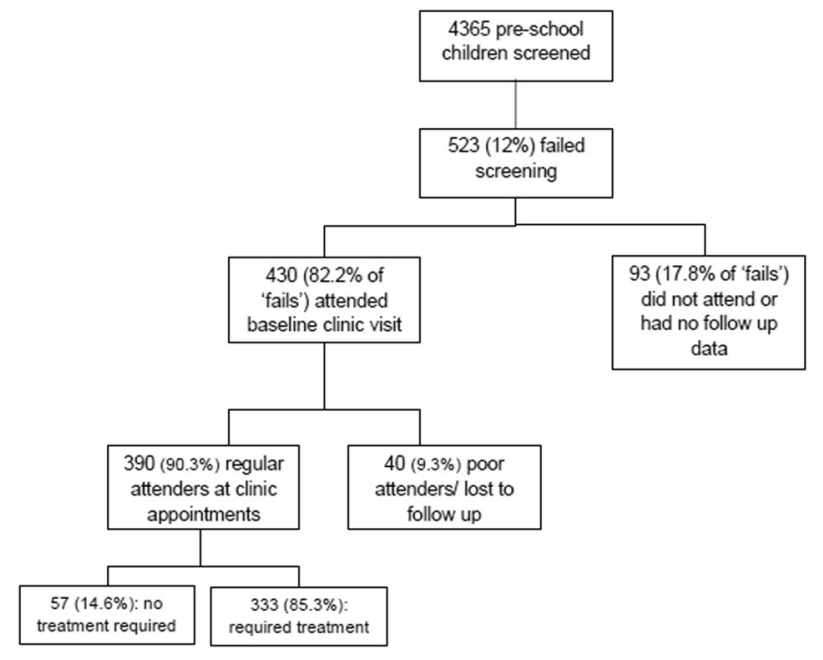

Fig. 1 Pre-School Visual Screening cohort in study period. Flow chart summarising the number of children who underwent pre-school visual screening (PSVS) and number of children included in the final analysis of this study.

two further appointments before being discharged via letter to their general practitioner (GP) and health visitor (HV). This is summarised in Fig. 1. Of the 430 children who were seen after the screening event, 40 failed to attend before treatment was completed. This group of children was categorised as poor attenders and their last recorded visual outcomes were used for a separate analysis.

\section{Definitions}

\section{Scottish index of multiple deprivation (SIMD)}

The SIMD 2012 (Scottish Government) is a multidimensional indicator, taking account of seven domain scores to produce an overall deprivation score for different postcodes. In our series of case studies, we have divided the SIMD into two distinct groups to examine the link between extreme deprivation and long-term visual outcomes: Quintile 1 (0-20\% most deprived) and Quintiles 2-5 (20-100\% least deprived).

\section{Health plan indicator (HPI)}

This is a unique code given by the assigned HV of every child in the UK based on a comprehensive assessment of the needs of children and individual family circumstances. Three HPI codes were used at the time of this study and they, in order of increasing need for input from health and social services are core (C), additional (A) and intensive (I). A child from a stable home with no concerns would be assigned 'Core' and receive HV and GP input; a child from an unstable home, for example with substance abuse problems, could be assigned 
'Intensive' and subsequently receive more input from health and social services. The HPI is the only formally applied measure of the stability and security of a child's home environment, it is widely used and well validated.

\section{Strabismus}

Full orthoptic assessment of strabismus was undertaken, strabismus included any constant or intermittent heterotropia, and micro-strabismus.

\section{Amblyopia}

We defined amblyopia as BCVA $\geq 0.2 \log$ MAR in the amblyopic eye and/or interocular difference of three or more $\log$ MAR lines. We excluded children with co-existing ocular abnormalities precluding normal vision. For children with bilateral amblyopia, visual acuity of the worse eye at baseline was used for comparison purposes.

We categorised amblyopia severity into three categories based upon the worse eye BCVA using the US Pediatric Eye Disease Investigator Group (PEDIG) definitions [14]; Mild: better than $0.3 \log$ MAR; Moderate: $0.3-0.7 \log M A R$; Severe: worse than $0.7 \log$ MAR.

\section{Binocular vision (BV)}

At the screening event, the orthoptists indicated "yes" or "no" for BV based on a child's response to a $15 \Delta$ prism reflex test and screening TNO plates. BV was further assessed at all clinic appointments. Frisby stereo-acuity test was used to assess stereopsis and Wirt fly was used if Frisby was not achieved. Motor fusion was assessed using the 15 or $20 \Delta$ base out test. When BV was not performed at the discharge visit, the final recorded BV closest to a child's discharge visit was used for comparison with the BV recorded at the first orthoptic visit which was subsequent to refraction and fundus check.

The range of BV was divided into three groups. Normal BV: Stereopsis better than $170 \mathrm{~s}$ of arc and the ability to overcome a prism; Poor BV: stereopsis of 170-600 s of arc irrespective of ability to overcome a prism or the inability to overcome a prism irrespective of level of stereopsis; No BV: Stereopsis poorer than $600 \mathrm{~s}$ of arc and the inability to overcome a prism.

\section{Statistical analysis}

SPSS statistical package (IBM SPSS Statistics for Windows, V.19.0, IBM Corp, Armonk, NY, USA) was used for data analyses. Chi-squared test $\left(\chi^{2}\right)$ was used to calculate the association between categorical variables and socioeconomic background as well as home circumstance based on SIMD and HPI, respectively. One-way analysis of variance was used to assess the difference in continuous variables among different subgroups. Hypothesis test of the equality of two proportions were used to compare proportions of amblyopia and BV. Mixed regression model was used to evaluate the relationship between BCVA and BV at discharge. All analyses were done with $95 \%$ confidence interval, and a $p$ value of $\leq 0.05$ considered statistically significant.

\section{Results}

\section{Study group and background demographics}

Results of the first clinic appointment (repeat orthoptic assessment, refraction and examination) were available for 430 of the 523 children (82.2\%) who failed screening. The remaining 93 of the 523 children (17.8\%) either did not attend their referral appointment from screening (Baseline visit) or there were no data available.

Of those who did attend their first appointment the attendance rate for follow-up at the eye clinic was $90.7 \%$ (390/430) (Fig. 1).

Background demographic and pattern of attendance to follow-up clinic visits are summarised in Table 1 .

\section{Poor attenders}

Forty of the 430 children (9.3\%) with follow-up results were categorised as poor attenders. Mean $( \pm$ standard deviation) age at discharge for this group was $6.2 \pm 1.2$ years old; their mean duration of follow-up was $26.5 \pm$ 10.5 months.

Sixteen $(40.0 \%)$ of the 40 poor attenders were from the 0-20\% most deprived socioeconomic group. The odds of children from the $0-20 \%$ most deprived socio economic group of having poor attendance were twice as high as for those from the 20-100\% least-deprived socioeconomic group (OR 2.19, 95\% CI 1.01-4.30, $p=0.003$ ).

Eighteen $(45.0 \%)$ of the 40 poor attenders were from a family assigned as either "intensive" (I) or "additional" (A). The odds of children from HPI groups I and A of attending poorly were four times higher than children from HPI group C (OR 3.94, 95\% CI 1.99-7.74, $p=0.002$ ).

\section{Ophthalmic diagnosis}

Of the remaining 390 children who were regular attenders, $387(99.2 \%)$ were discharged from the clinic after a mean follow-up time of $19.7 \pm 5.8$ months.

Twenty six of the 430 children $(6.0 \%)$ who met the referral criteria were discharged after one to two visits if their vision proved to be normal, these children were 
Table 1 This table details the background socioeconomic status, health plan indicator and pattern of attendance to follow-up clinic for study population.

\begin{tabular}{lc}
\hline & $\begin{array}{l}\text { No. }(\%) \text { of children } \\
(n=430)\end{array}$ \\
\hline Scottish index of multiple deprivation (SIMD) & \\
Quintile 1 (Most deprived) & $107(24.9 \%)$ \\
Quintile 2 & $62(14.4 \%)$ \\
Quintile 3 & $82(19.1 \%)$ \\
Quintile 4 & $130(30.2 \%)$ \\
Quintile 5 (Least deprived) & $49(11.4 \%)$ \\
Health Plan Indicator (HPI) & \\
Intensive (I) & $22(5.1 \%)$ \\
Additional (A) & $63(14.7 \%)$ \\
Core (C) & $345(80.2 \%)$ \\
Attendance & \\
Regular attender & $390(90.7 \%)$ \\
Poor attender & $40(9.3 \%)$ \\
Gender & \\
Male & $207(48.1 \%)$ \\
Female & $223(51.9 \%)$ \\
Ethnicity & \\
Caucasian & $421(97.9 \%)$ \\
Others & $9(2.1 \%)$ \\
\hline
\end{tabular}

classed as false positives and excluded from the outcome data. A further $31(7.2 \%)$ children were reviewed at least three times without any active intervention because they had reduced vision but no evidence of refractive error or pathology and eventually they demonstrated a satisfactory level of vision $(\mathrm{VA}<\log \mathrm{MAR}$ 0.2). These children were grouped as "visually immature" because with age and repeated practice at the assessment they were able to achieve normal vision. These children underwent cycloplegic refraction and dilated fundoscopy by a paediatric ophthalmologist or hospital optometrist, as all our children do, and no pathology was found.

\section{Management}

Two hundred and fifty-four children were prescribed glasses; this was the sole intervention for 173 of the 390 children $(44.4 \%)$ who attended regularly. One hundred and two $(26.1 \%)$ were treated with occlusion. Six children $(1.5 \%)$ received atropine penalisation, four of whom had adjuvant patching. Two refused patching.

A total of twenty-four $(6 \%)$ children were recorded as being noncompliant with either glasses $(n=4)$ or occlusion $(n=20)$, of which ten were poor attenders and were lost to follow-up. Sixteen $(66.7 \%)$ of these children were from a family assigned as "intensive" or "additional". (OR 9.97,
95\% CI $0.23-0.71, p<0.001)$. Five $(20.8 \%)$ were from the $0-10 \%$ most socioeconomically deprived background.

Ten children $(2.1 \%)$ received surgical correction for strabismus, for whom the mean overall length of follow-up in total was $3.08 \pm 1.40$ years.

\section{Amblyopia}

The proportion of children with amblyopia at baseline and the final visit for both poor and regular attenders is shown in Fig. 2.

At baseline visit, 373 children (92.3\%) had amblyopia. In total, 62/373 (16.6\%) were categorised as mild, 273/373 (73.1\%) moderate and 38/373 (10.2\%) severe.

For poor attenders $(N=40)$ who were lost to follow-up, $72.5 \%$ had their last-measured BCVA recorded as meeting the amblyopia threshold; of these $6(15.0 \%)$ were categorised as mild, $20(50.0 \%)$ moderate and $3(7.5 \%)$ severe.

For the remaining 364 children who attended clinic regularly, $70.9 \%$ children $(n=258)$ had BCVA better than $0.2 \log$ MAR at discharge. Difference between the proportion of children with amblyopia at baseline and at discharge was statistically significant $(p<0.001)$.

The odds of having amblyopia at the baseline clinic visit were 29 times higher than at the point of discharge (OR $29.29,95 \%$ CI $7.84-26.14, p<0.001)$. The odds of having residual amblyopia for poor attenders was significantly higher than children who attended follow-up appointments regularly (OR 6.42, 95\% CI 4.25-10.56, $p<0.001$ ).

\section{Binocular vision}

At the point of screening 161 of 430 children (37.4\%) who were referred had their BV recorded as "no". At baseline orthoptic clinic visit, after refraction and fundus examination, 118/404 (29.2\%) had good BV, 185/404 (45.8\%) had poor BV and 101/404 (25.0\%) had no BV. Of the regular

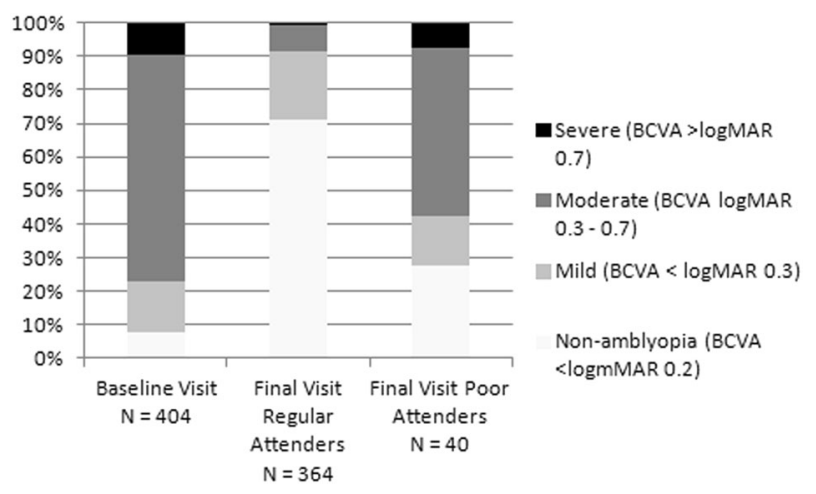

Fig. 2 Vision outcomes versus attendance. This graph shows the distribution of amblyopia based on the level of severity (mild, moderate and severe) at baseline and final visit for regular and poor attenders. 


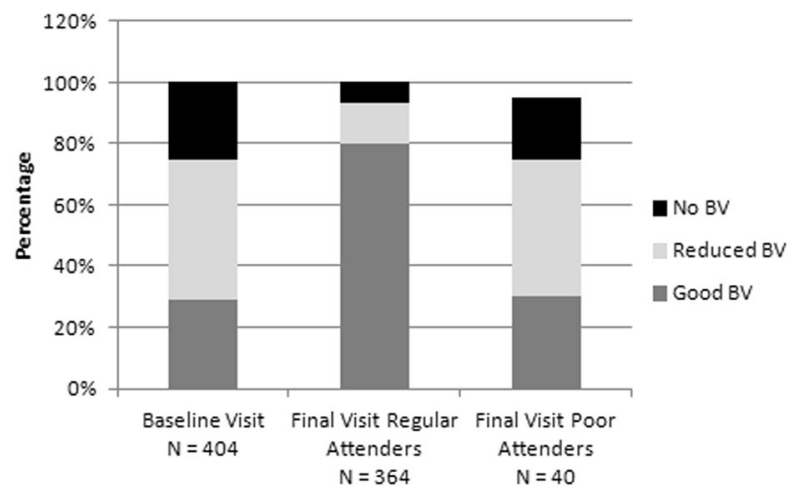

Fig. 3 Binocular vision outcomes versus attendance. This graph shows the distribution of binocular vision (BV) at baseline and final visit for regular and poor attenders.

attenders, at discharge, 291/364 (79.9\%) had good BV, 49/364 (13.5\%) had poor BV and 24/364 (6.6\%) had no $\mathrm{BV}$. The distribution of $\mathrm{BV}$ pattern proportion at baseline and at the final visit is summarised in Fig. 3.

The difference between the proportion of children with good BV at baseline and at discharge was statistically significant $(p<0.001)$. The odds of having good $\mathrm{BV}$ at discharge for the regular attenders was seven times higher than that at baseline (OR 9.7, 95\% CI 0.62-1.10, $p<0.001$ ). There was a positive association between BCVA and BV at final discharge $(r=0.88,95 \%$ CI $0.76-0.91, p<0.001)$.

Of the 40 poor attenders, at baseline clinic visit, $8(21.1 \%)$ had good BV, 21 (55.3\%) had poor BV and 9 (23.7\%), had no BV. Twelve (31.6\%) were last recorded as having good BV, 18 (47.4\%) had poor BV and 8 (21.1\%) had no BV.

The difference between the proportion of children having poor/no BV among the poor attenders compared to the regular attenders is significant $(p<0.001)$.

\section{Comparison of final visual outcome based on SIMD and $\mathrm{HPI}$}

The relationship between socioeconomic background (SIMD), home circumstance as indicated by HPI and adverse visual outcome for children who attended well ( $n=$ 364) was examined (Table 2). Results were independent of gender and ethnicity for these children.

There was no statistical difference in the odds of children from the 0-20\% most deprived socioeconomic background having poorer visual outcomes (final BCVA worse than $\log$ MAR 0.2, improvement of BCVA less than logMAR 0.2 and poor or no BV) compared to children from the 20-100\% least-deprived socioeconomic background. ( $p=0.745, p=0.710, p=0.219$, respectively).

However, children from HPI groups I and A were five times more likely to have a final BCVA worse than 0.2 $\operatorname{logMAR}$ (OR 5.37, 95\% CI 3.29-10.07, $p<0.001$ ) and three times more likely to have poor or no BV (OR 3.41,

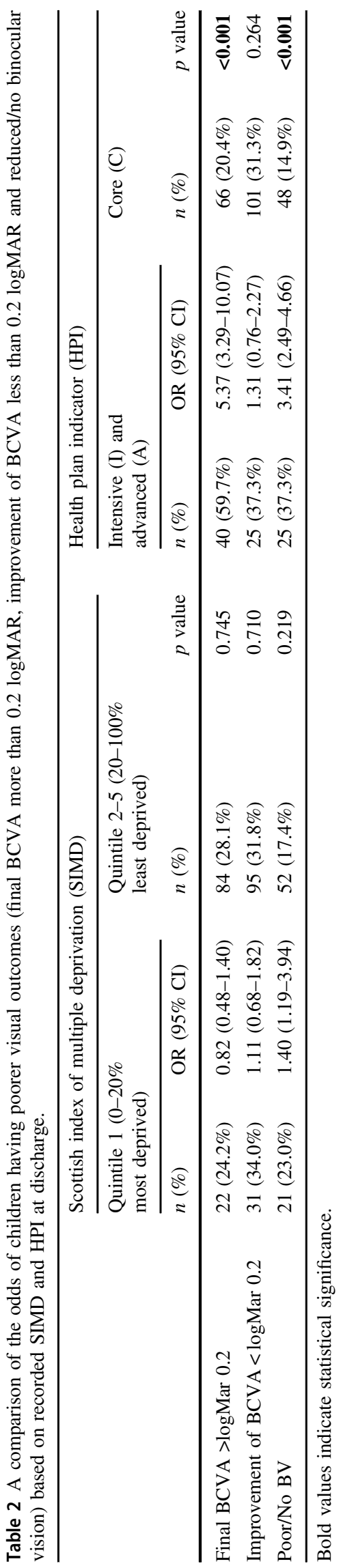


95\% CI 2.49-4.66, $p<0.001)$ compared to children from a family assigned as "Core".

\section{Discussion}

Overall the children in our real-life cohort responded well to amblyopia treatment, with $70.9 \%$ of good attenders achieving a BCVA of better than $0.2 \log$ MAR and $61.7 \%$ achieving an improvement of at least $0.2 \log$ MAR. The proportion of children with moderate to severe amblyopia reduced from $77.0 \%$ at baseline to $8.7 \%$ at discharge. The magnitude of this improvement was comparable to that observed in randomised controlled trials such as the ALSPAC and PEDIG studies [15, 16].

Our results also demonstrated an increase in the proportion of children with good BV from $29.2 \%$ at baseline to $79.9 \%$ at discharge. Previous studies have shown that BV can improve following treatment of amblyopia [17-19]. Our study supports these findings, including in those who had intermittent heterotropias and micro-strabismus.

This study found that children from more deprived socioeconomic backgrounds and those from families requiring more social care input (HPI) are more likely to have poor attendance. Analysis of the visual outcomes for poor attenders in our study showed that they were six times more likely to have residual amblyopia and almost ten times more likely to have poor or no BV compared to regular attenders. Children who were poor attenders and those who became lost to follow-up record a relatively earlier last visit during their treatment, which meant they had fewer attempts to have improved visual acuity and less time to be treated in a closely monitored specialist setting. It is possible that poorer health seeking behaviour among parents who require social care input adversely impacts on the attendance rate of their children as they are less likely to engage with health services [20]. The attendance rate for follow-up eye clinic appointments in our study sits around $90.7 \%$, which is higher than most other studies [15, 21].

Our results have demonstrated that irrespective of a child's socioeconomic background, with regular follow-up, intensive treatment and good compliance, children from more deprived backgrounds have similarly good visual outcomes compared to less deprived children. This is an important finding as our initial study found that children who were from deprived backgrounds were more likely to fail screening [13]. In this study, children from less stable home circumstances who required "Intensive" and "additional" support were 4.5 times more likely to have a worse final BCVA and three times more likely to have poor or no BV compared to children from the "core" group. This study also reported a similar association between worse home circumstances and screening outcomes [13]. Children from the "Intensive" and "additional" group were ten times more likely to be treatment non-compliant, irrespective of socioeconomic background.

The reasons for this difference in screening failure rates are not known but it has been theorised that poorer prenatal/ antenatal care [22, 23] associated with increased rates of maternal smoking, alcohol and drug intake [24-27] which are commoner in deprived areas [28] may be significant contributors. This current study suggests that, if these factors are indeed relevant, they are reversible with adequate treatment. Comprehensive screening to pick up these most vulnerable children is essential and it must be followed up by methods to encourage treatment compliance.

One limitation of our study was the retrospective nature of the data collection, but the benefit of this methodology is that the observational findings are representative of the reallife situation. The percentage of children lost to follow-up $(9.3 \%)$ was slightly higher than other studies $[11,15,16]$. However, our results have shown that the majority of the poor attenders were from more socioeconomically deprived and adverse family backgrounds and that the home circumstances associated with poor attendance have the most impact on the outcome. Hence although this is a form of bias, it contributes to a possible underestimation of the negative impact of deprivation on the final visual outcome.

This study reports the treatment and visual outcomes of a whole-population orthoptic-delivered pre-school visual screening service. It identified that attendance is the key to the final visual outcome for children; children from deprived/high risk homes were much more likely to not attend appointments and did not do well. It is crucial for children who are already being brought up in a challenging environment that the screening system supports them and their families, in order that they may have the same successful outcomes as their more fortunate peers.

\section{Summary}

\section{What was known before}

- Pre-school visual screening is effective in identifying children at risk of amblyopia and is recommended to be offered for all children aged 4 to 5 years.

- Children who are socioeconomically deprived and those who come from homes that require high levels of social care input are more likely to fail visual screening.

\section{What this study adds}

- Long-term outcomes of orthoptic-delivered pre-school visual screening demonstrate no difference in bestcorrected visual acuity (BCVA) and/or binocular vision 
(BV) outcomes based on socioeconomic deprivation alone-compliance with hospital attendance rates is more critical.

- Children from homes where extra social care support is required attend less well and are more likely to have poorer long-term visual outcomes.

Author contributions Data were collected by YNN, UOC and CG. Statistical analysis was performed by $\mathrm{YN}$. YN produced the initial draft manuscript, all authors contributed to the revision and UOC and CJM prepared the final draft.

\section{Compliance with ethical standards}

Conflict of interest The authors declare that they have no conflict of interest.

Ethical approval Ninewells Hospital Orthoptic and Ophthalmology departments have ongoing Caldicott guardianship approval for analysis and review of pre-school vision screening outcomes.

Publisher's note Springer Nature remains neutral with regard to jurisdictional claims in published maps and institutional affiliations.

\section{References}

1. Grant S, Moseley MJ. Amblyopia and real-world visuomotor tasks. Strabismus. 2011;19:119-28.

2. Levi DM, Knill DC, Bavelier D. Stereopsis and amblyopia: a mini-review. Vis Res. 2015;114:17-30.

3. Powell C, Hatt SR. Vision screening for amblyopia in childhood. Cochrane Database Syst Rev. 2009:CD005020.

4. Carlton J, Karnon J, Czoski-Murray C, Smith KJ, Marr J. The clinical effectiveness and cost-effectiveness of screening programmes for amblyopia and strabismus in children up to the age of 4-5 years: a systematic review and economic evaluation. Health Technol Assess. 2008;12:iii, xi-194.

5. Taylor K, Elliott S. Interventions for strabismic amblyopia. Cochrane Database Syst Rev. 2014:Cd006461.

6. Taylor K, Powell C, Hatt SR, Stewart C. Interventions for unilateral and bilateral refractive amblyopia. Cochrane Database Syst Rev. 2012:Cd005137.

7. Espinosa JS, Stryker MP. Development and plasticity of the primary visual cortex. Neuron. 2012;75:230-49.

8. Committee NS. The UK NSC policy on vision defects screening in children. 2013. http://legacyscreening.phe.org.uk/vision-child.

9. Hall DMB, editor. Health for all children. Oxford: Oxford University Press; 2006.

10. Toufeeq A, Oram AJ. School-entry vision screening in the United Kingdom: practical aspects and outcomes. Ophthalmic Epidemiol. 2014;21:210-6.

11. Solebo AL, Cumberland PM, Rahi JS. Whole-population vision screening in children aged 4-5 years to detect amblyopia. Lancet. 2015;385:2308-19.

12. Schmucker C, Grosselfinger R, Riemsma R, Antes G, Lange S, Lagreze W, et al. Effectiveness of screening preschool children for amblyopia: a systematic review. BMC Ophthalmol. 2009;9:3.

13. O'Colmain U, Low L, Gilmour C, MacEwen CJ. Vision screening in children: a retrospective study of social and demographic factors with regards to visual outcomes. Br J Ophthalmol. 2016;100: 1109-13.

14. Repka MX, Kraker RT, Holmes JM, Summers AI, Glaser SR, Barnhardt $\mathrm{CN}$, et al. A randomized trial of atropine vs. patching for treatment of moderate amblyopia in children. Arch Ophthalmol. 2002;120:268-78.

15. Williams C, Northstone K, Harrad RA, Sparrow JM, Harvey I. ALSPAC Study Team. Amblyopia treatment outcomes after screening before or at age 3 years: follow up from randomised trial. BMJ. 2002;324:1549.

16. Pediatric Eye Disease Investigator Group. Pharmacological plus optical penalization treatment for amblyopia: results of a randomized trial. Arch Ophthalmol. 2009;127:22-30.

17. Awadein A, Fakhry MA. Changes in binocular function in anisometropic nonstrabismic children with optical correction and occlusion therapy. J AAPOS. 2011;15:545-50.

18. Cleary M, Houston CA, McFadzean RM, Dutton GN. Recovery in microtropia: implications for aetiology and neurophysiology. Br J Ophthalmol. 1998;82:225-31.

19. Lee SY, Isenberg SJ. The relationship between stereopsis and visual acuity after occlusion therapy for amblyopia. Ophthalmology. 2003;110:2088-92.

20. MacKian S. A review of health seeking behaviour: problems and prospects. Manchester: Health Systems Development Programme, University of Manchester; 2003.

21. Williams C, Northstone K, Harrad RA, Sparrow JM, Harvey I. ALSPAC Study Team. Amblyopia treatment outcomes after preschool screening $\mathrm{v}$ school entry screening: observational data from a prospective cohort study. Br J Ophthalmol. 2003;87: 988-93.

22. Tarczy-Hornoch K, Varma R, Cotter SA, McKean-Cowdin R, Lin $\mathrm{JH}$, Borchert MS, et al. Risk factors for decreased visual acuity in preschool children: the multi-ethnic pediatric eye disease and Baltimore pediatric eye disease studies. Ophthalmoloy. 2011;118: 2262-73.

23. Gilbert CE, Ellwein LB. Refractive Error Study in Children Study Group. Prevalence and causes of functional low vision in school age children: results from standardized population surveys in Asia, Africa and Latin America. Investig Ophthalmol Vis Sci. 2008;49: 877-81.

24. Chew E, Remaley NA, Tamboli A, Zhao J, Podgor MJ, Klebanoff M. Risk factors for esotopia and exotropia. Arch Ophthalmol. 1994;112:1349-55.

25. Hakim RB, Tielsch JM. Maternal cigarette smoking during pregnancy: a risk factor for childhood strabismus. Arch Ophthalmol. 1992;110:1459-62.

26. McGlone L, Hamilton R, McCulloch DL, MacKinnon JR, Bradnam $\mathrm{M}$, Mactier $\mathrm{H}$. Visual outcome in infants born to drugmisusing mothers prescribed methadone in pregnancy. $\mathrm{Br} \mathbf{J}$ Ophthalmol. 2014;98:238-45.

27. Spiteri Cornish K, Hrabovsky M, Scott NW, Myerscough E, Reddy AR. The short and long-term effects on the visual system of children following exposure to maternal substance misuse in pregnancy. Am J Ophthalmol. 2013;156:190-4.

28. Davi-Gray A, Moor S, Spencer C, Woodward LJ. Psychosocial characteristics and poly-drug use of pregnant women enrolled in methadone maintenance treatment. Neurotoxicol Teratol. 2013;38: $46-52$. 